\title{
Kinesiophobia Relates to Decreased Sports Capability Perceptions, and Altered Gait Following ACL Reconstruction
}

\author{
M. R. Goh'1, Y. H. D. Lee ${ }^{2}$, C. C. R. Teo ${ }^{2}$, J. Nyland ${ }^{3}$ \\ 1 Department of Rehabilitation, Woodlands Health Campus, Singapore \\ 2 Department of Orthopedic Surgery, Changi General Hospital, Singapore \\ 3 Kosair Charities College of Health and Natural Sciences, Spalding University, Louisville (KY), U.S.A.
}

\section{CORRESPONDING AUTHOR:}

John Nyland

Kosair Charities College of Health and

Natural Sciences

Spalding University

901 S. Fourth Street

Louisville (KY), U.S.A.

E-mail: jnyland@spalding.edu

DOI:

10.32098/mltj.04.2021.11

LEVEL OF EVIDENCE: 2B

\begin{abstract}
SUMMARY
Introduction. Kinesiophobia can negatively influence perceived knee function after anterior cruciate ligament reconstruction (ACLR). Less is known about its influence on perceived sports capability, and gait kinematics.

Methods. At 3 months after ACLR, 34 male patients completed the Tampa Scale of Kinesiophobia (TSK). Raw and grouped scores (group $1=$ high kinesiophobia; group 2 = low kinesiophobia) were compared with perceived sports capability and gait kinematics over the initial 9 months after ACLR. The Tegner Activity Level Scale (TALS) was completed pre-injury, before ACLR, and at 6 and 9 months after ACLR. The Lysholm Knee Scoring Scale was completed pre-surgery, and at 6 and 9 months after ACLR. Walking and running gait analysis was performed at 3 and 6 months; and 6 months after ACLR, respectively $(P \leq 0.05)$. Results. Group TALS scores were similar pre-injury, however, Group 1 had lower TALS scores pre-surgery, and at 6 and 9 months after ACLR. Inverse relationships were observed for the 3 month TSK score and TALS scores before ACLR, and at 6 and 9 months afterwards. By 9 months following ACLR, kinesiophobia remained related to sports capability. Kinesiophobia had a stronger influence on side-to-side gait asymmetries than surgical side for hip adduction and mid-stance knee flexion when walking at 3 months after ACLR, for mid-stance knee flexion and terminal stance knee extension when walking at 6 months after ACLR, and for terminal stance knee extension when running at 6 months after ACLR. Conclusions. High kinesiophobia following ACLR was related to walking and running gait kinematic characteristics and sports capability perceptions.
\end{abstract}

KEY WORDS

Knee joint; biomechanics; activity level; sports capability; fear avoidance.

\section{INTRODUCTION}

For athletically active patients, the primary reason for undergoing ACL reconstruction (ACLR) is to safely return to pre-injury sports participation (1). However, widely ranging return to sport rates (43\%-93\%) have been reported after ACL reconstruction (2-5). Successful return to sports after ACLR is influenced by multiple physical and psychological factors (6-8). Ardern et al. (2) reported that knee function at one-year after ACLR was not related to ultimate return to sports success. By 2 and 5 years after ACLR, Eriksson et al. (9) reported that neuromuscular, balance, and proprioception impairments continued to negatively influence lower extremity movements. Suboptimal neuromuscular recovery after ACLR is a contributing factor to increased ipsilateral knee re-injury or contralateral knee injury rates (10-12).

"Kinesiophobia" exists when a patient perceives excessive, irrational, and debilitating physical movement and activity fear due to perceived painful injury or re-injury vulnerability 
(13). The Tampa Scale for Kinesiophobia (TSK) is a valid, reliable, and responsive kinesiophobia or movement fear perception measurement (13). This patient-reported questionnaire has a scoring range from 17-68 where higher scores indicate greater kinesiophobia (14). A TSK score of $>37$ represents high kinesiophobia (15). The Lysholm Knee Scoring Scale, and the Tegner Activity Level Scale (TALS) are widely used patient-administered surveys for measuring perceived knee function, and sports capability, with minimal detectable change (MDC) values of 8.9, and 1, respectively (16-18).

An essential early rehabilitation plan component after ACLR is gait training. In a biomechanical comparison of 8 patients at 6 months following bone-patellar tendon-bone (BPTB) autograft ACLR, and 22 healthy subjects, Devita et al. (3) reported that patients had normal walking kinematics, however, stance phase hip extensor torque was increased to compensate for reduced knee extensor torque. Using kinematic and electromyographic methods to compare 25 patients with ACL deficiency prior to, and at 6 weeks, 4 months, 8 months and 12 months following BPTB autograft ACLR with 51 healthy subjects, Knoll et al. (4) reported that pre-injury walking gait kinematic restoration required at least 8 months.

While gait asymmetries are known to persist following ACLR, their relationship to kinesiophobia, knee function, and sports capability perceptions have not been fully elucidated. The purpose of this pilot study was to determine if patients with high kinesiophobia measured at the end of the acute care ACLR rehabilitation phase ( 3 months) had different walking ( 3 and 6 months) and running (6 months) gait kinematics, perceived knee function, and sports capability compared to patients with low kinesiophobia over the initial 9 months after ACLR.

\section{MATERIALS AND METHODS}

\section{Subject selection}

Following hospital medical ethics committee approval (SingHealth Institutional Review Board, Singapore), 34 consecutive male patients from a single institution who were about to undergo primary ACLR were recruited for study participation. Each subject provided written informed consent. All subjects had complied with the rehabilitation protocol. Revision ACLR, multi-ligament knee injury, subjects who displayed a grade 3, "explosive" pivot shift test prior to ACLR, or patients with co-morbidities other than partial meniscectomy or repair, or articular cartilage debridement were excluded from study participation (figure 1).

\section{Surgical procedure}

Femoral and tibial tunnels were individually prepared. All subjects received a four-strand hamstring autograft with

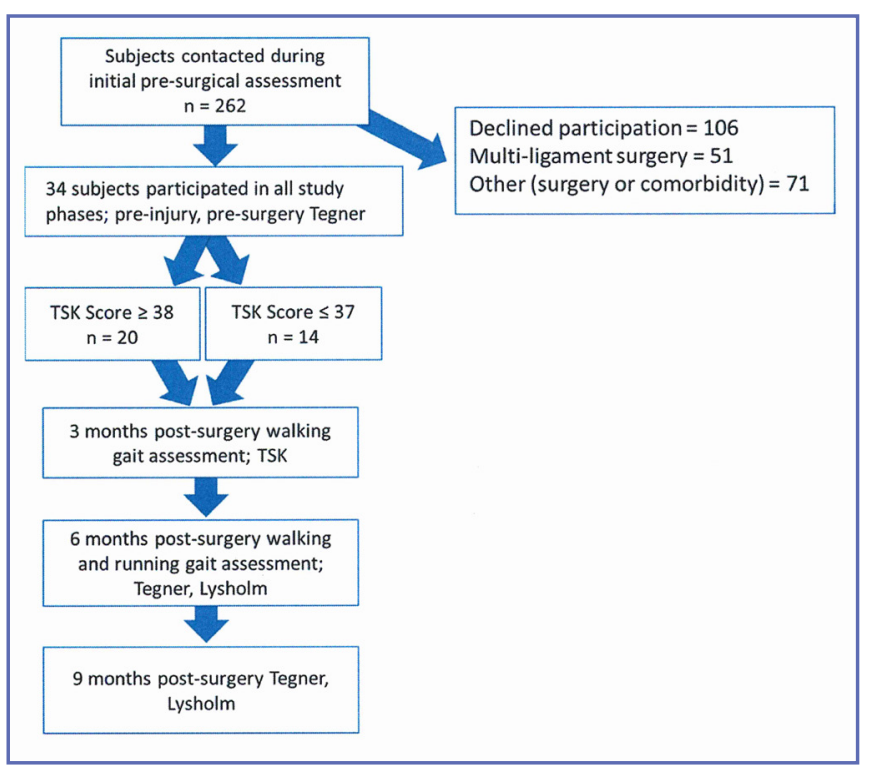

Figure 1. Subject recruitment and assessment progression. TSK: Tampa Scale of Kinesiophobia.

cortical femoral fixation and tibial interference screw fixation with a back-up staple.

\section{Rehabilitation protocol}

Over the first year following ACLR, patients participated in a standardized, three phase rehabilitation protocol: Acute care (0-3 months), strength training (4-6 months), and return to sports training (7-9 months). The protocol was adapted from previously reported guidelines $(19,20)$. Full release to unrestricted sports generally occurred near the end of the $9^{\text {th }}$ post-surgical month. Most subjects initiated full weight bearing (FWB) walking during the first week after ACLR. Subjects who underwent concomitant meniscal repair or articular cartilage debridement initiated FWB 4 weeks following ACLR. Isometric quad-setting, and four-way progressive resistance straight leg raises were initiated during the first week. Mini-squats, and progressive resistance multi-directional lunges were added after FWB was initiated. At 3 months after ACLR, progressive resistance seated knee extension, hamstring curls, and single leg pressing was initiated. Stationary cycling, and elliptical device training were started at 3 weeks, and 8 weeks after ACLR, respectively. A jog-to-run progression was initiated at 4-5 months, when the surgical lower extremity achieved $80 \%, 10$ repetition maximum bilateral resistance equivalency with the non-surgical lower extremity for seated knee extension (knee extensors), hamstring curls (knee flexors), and single leg press (composite lower extremity extension). 
At 6 months following ACLR, progressive resistance hip abduction, single leg hip thrusts, hip extensions, squats, lunges, and neuromuscular core exercises were added. At 8 months after ACLR, progressive intensity plyometric jumping, and sudden directional change agility tasks were added. The study surgeon assessed knee laxity at 3, 6 and 9 months after ACLR using manual maximum Lachman and pivot shift tests. All subjects displayed normal $(\leq 2 \mathrm{~mm})$ anterior translational knee laxity and negative pivot shift tests over the course of the study.

\section{Kinematic gait analysis}

Two-dimensional walking $(4 \mathrm{~km} / \mathrm{hr}=24 \mathrm{~min} /$ mile pace $)$, and running $(8 \mathrm{~km} / \mathrm{hr}=12 \mathrm{~min} /$ mile pace $)$ kinematic gait analysis were assessed on a treadmill with digital video cameras placed on the same side of the treadmill as the surgical lower extremity (1.8 $\mathrm{m}$ away) facing the center (sagittal, camera \#1), and at each end of the treadmill (anterior $=0.5 \mathrm{~m}$ away) facing the center (frontal, cameras \#2) for motion capture $(60 \mathrm{~Hz})$ (SiliconCoach ${ }^{\mathrm{TM}}$ System, Tarn Group, Dunedin, New Zealand). With subjects wearing cycling shorts, $2.54 \mathrm{~cm}$ diameter skin markers with adhesive backing were placed on the shorts over the bilateral anterior (ASIS) and posterior (PSIS) superior iliac spines, the greater trochanter, lateral knee joint line, patellar center, and lateral malleolus of each lower extremity. After a 3 minute warmup at volitional walking pace, a $30 \mathrm{sec}$ duration data collection was performed for each condition.

Lower extremity joint displacements during gait were determined from the following angles.

- Camera \#1: hip flexion-extension = midway point along the line between the ipsilateral ASIS-PSIS and the great- er trochanter-mid-lateral knee joint line; knee flexion-extension $=$ greater trochanter, mid-lateral knee joint line, lateral malleolus.

- Camera \#2: hip abduction/adduction = intersection between the line formed by the bilateral ASIS, the stance lower extremity ASIS-patellar center. Two-dimensional kinematic joint angle measurements were performed by the primary investigator using the software cursor function (figure 2). Good measurement reliability has been reported using this system for the kinematic analysis of sports movement performance (21-23), and good-to-excellent reliability was identified for this study during pilot testing.

\section{Self-reported patient outcome surveys}

The Tampa Scale of Kinesiophobia (TSK) was administered at 3 months following ACLR at the end of the acute care rehabilitation phase (6-8). The Lysholm Knee Scoring Scale was administered before ACLR, 6 months (beginning of return to sports training phase), and 9 months (return to play decision-making phase) after ACLR. Patients were asked to retrospectively self-report their pre-injury TALS score based on perceived sports capability prior to sustaining the index ACL injury. Pre-surgery, 6 months (beginning of return to sports training phase), and 9 months (return to play decision-making phase) after ACLR TALS scores were collected as subjects self-reported their current highest sports activity level capability.

\section{Statistical analysis}

Shapiro-Wilk tests revealed data normality, therefore, parametric statistical tests were performed for continuous

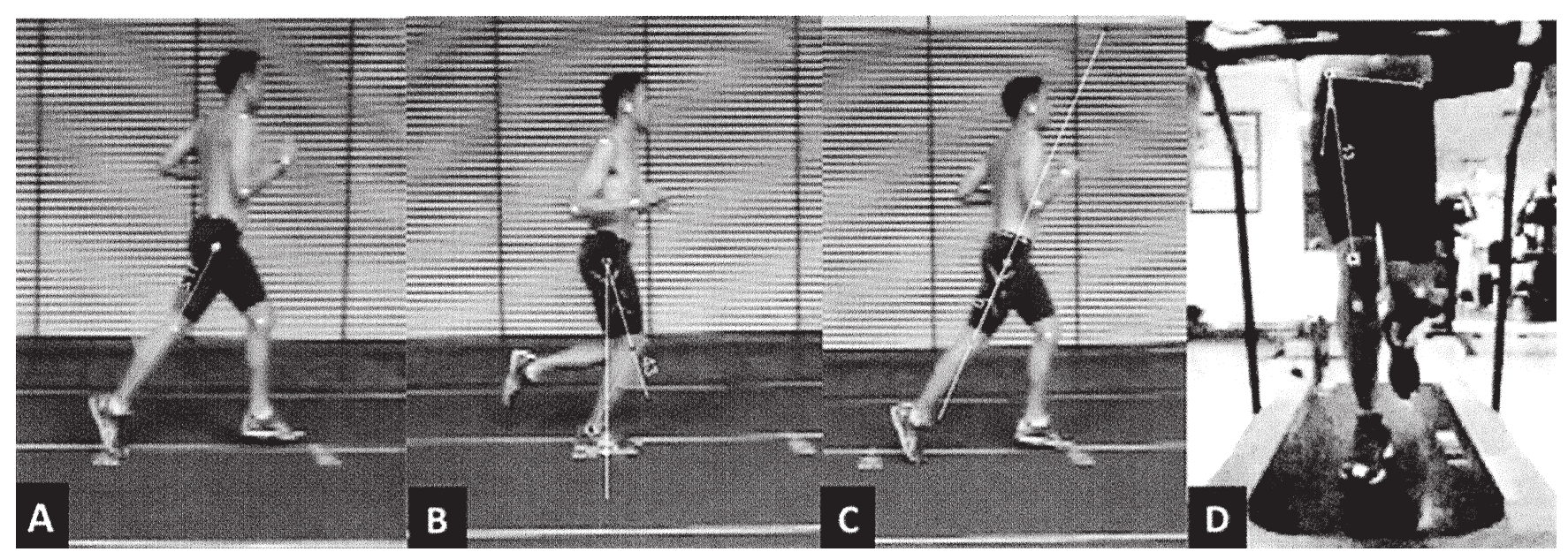

Figure 2. Two-dimensional gait analysis reference points. Knee extension at terminal stance (A), knee flexion at midstance (B), hip extension at terminal stance (C), hip adduction at midstance (D). 
data. A series of mixed model, two-way ANOVA (Kinesiophobia group, Side) were used to compare gait variables. Dependent sample t-tests were used to compare Lysholm Knee Scoring Scale score group differences. Chi-square tests were used to evaluate group frequency differences for the number of subjects who underwent concomitant partial meniscectomy, meniscal repair, or articular cartilage debridement, and to identify group TALS score response frequency differences. Pearson product moment correlations were used to more accurately delineate relationship strength between gait kinematic, and patient self-reported survey data (24). Statistical analysis was performed using SPSS ver. 26.0 software (SPSS-IBM, Armonk, NY, USA). An alpha level of $\mathrm{p} \leq 0.05$ was selected to indicate statistical significance.

\section{RESULTS}

All subjects completed each study component. Overall subject age was $24.7 \pm 6.9$ years and body mass index (BMI) was $26.3 \pm 6.9 \mathrm{~kg} / \mathrm{m}^{2}$. Kinesiophobia group subject demographics are displayed in table I. Although the high kinesiophobia group had greater Lysholm Knee Scoring Scale scores pre-injury compared to the low kinesiophobia group $(64.2 \pm 11.5$ vs $57.1 \pm 17.6, \mathrm{P}=0.03)$, this value did not meet the previously reported 8.9 MDC value (18), and statistically significant differences were not observed at 6 months (78.3 \pm 15.8 vs $82.3 \pm 15.6, \mathrm{P}=0.32)$, and 9 months $(84.7 \pm$ 14.2 vs $81.1 \pm 13.9, \mathrm{P}=0.92$ ) after ACLR.

Pre-injury TALS scores failed to reveal kinesiophobia group differences with both groups displaying scores indicating perceived recreational running, soccer, rugby or basketball playing capability. At pre-surgery, and at 6 and 9 months after ACLR the high kinesiophobia group had lower TALS scores with each group difference exceeding the one point MDC (18). Chi-square tests revealed that the high kinesiophobia group had significantly lower (3 vs $5, \mathrm{P}=0.03$ ) pre-surgery TALS score frequencies suggesting that they only perceived light labor, or sports swimming capability, compared to the low kinesiophobia group who perceived heavy labor, competitive cycling, or recreational jogging capability. At 6 months following ACLR, TALS score frequencies remained lower in the high kinesiophobia group ( 4 vs $7, \mathrm{P}=0.04$ ) suggesting that high kinesiophobia group subjects only perceived moderate labor, recreational cycling, or jogging capability, compared to the low kinesiophobia group who perceived the ability to play competitive tennis, running or handball, and recreational soccer, rugby, basketball or running. By 9 months after ACLR, TALS scores remained lower ( 6 vs $8, \mathrm{P}=0.04$ ) in the high kinesiophobia group suggesting perceived sports capability of only being able to safely participate in recreational tennis, badminton, handball or jogging, compared to the low kinesiophobia group who perceived the ability to safely participate in competitive badminton, track and field athletics (including jumping), or racquetball.

Correlation analysis failed to identify significant relationships between Lysholm Knee Scoring Scale scores, kinematic gait measurements, or TSK scores. However, correlation analysis revealed consistently significant moderate-to-low inverse relationships between the 3 month TSK, and each TALS score (figure 3). Over the 9 month time period following ACLR, the TSK score measured at 3 months after ACLR (end of the acute rehabilitation phase) continued to influence sports activity capability perceptions (figure 4).

At 3 months following ACLR, walking kinematic gait analysis revealed that the high kinesiophobia group had greater peak stance phase hip adduction, and peak mid-stance phase knee flexion than the low kinesiophobia group (table II). At 6 months after ACLR, the high kinesiophobia group continued to display greater peak mid-stance phase knee

Table I. Subject demographics.

\begin{tabular}{|c|c|c|c|c|}
\hline & & $\begin{array}{l}\text { Group } 1 \\
\text { (High TSK) }\end{array}$ & $\begin{array}{l}\text { Group } 2 \\
\text { (Low TSK) }\end{array}$ & $\mathbf{P}$ \\
\hline \multirow{3}{*}{ Study Entry } & Age (years) & $25.3 \pm 6.9$ & $24.0 \pm 7.0$ & 0.61 \\
\hline & BMI $(\mathrm{kg} / \mathrm{m} 2)$ & $26.5 \pm 8.0$ & $26.1 \pm 5.0$ & 0.88 \\
\hline & $\begin{array}{l}\text { \# Partial Meniscectomy/Meniscus Repair Articular } \\
\text { Cartilage debridement }\end{array}$ & $3 / 2 / 3$ & $2 / 2 / 2$ & 0.64 \\
\hline
\end{tabular}




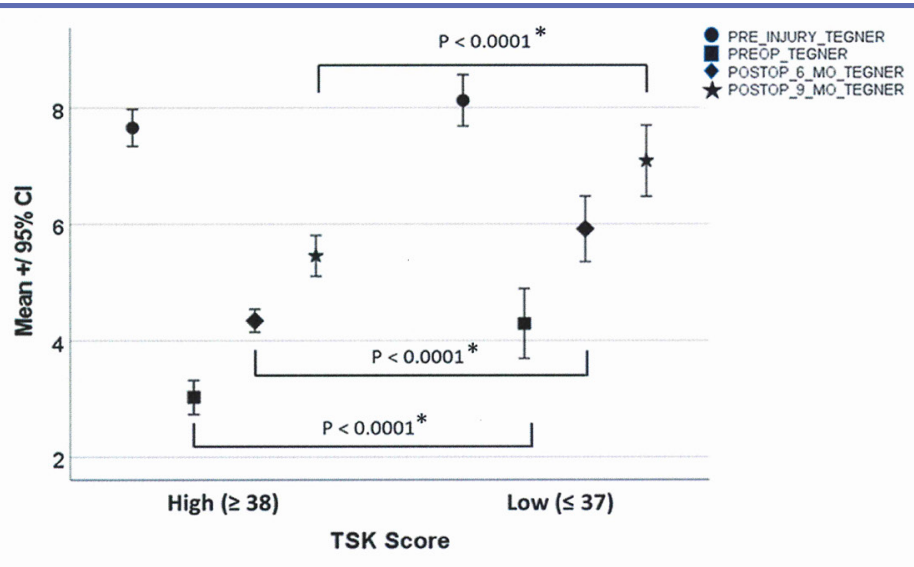

Figure 3. Although groups displayed comparable Tegner Activity Level Scale scores prior to the index ACL injury, the high kinesiophobia group had significantly lower scores prior to surgery and over the 9 month rehabilitation program.

TSK: Tampa Scale of Kinesiophobia; *p $\leq 0.05$.

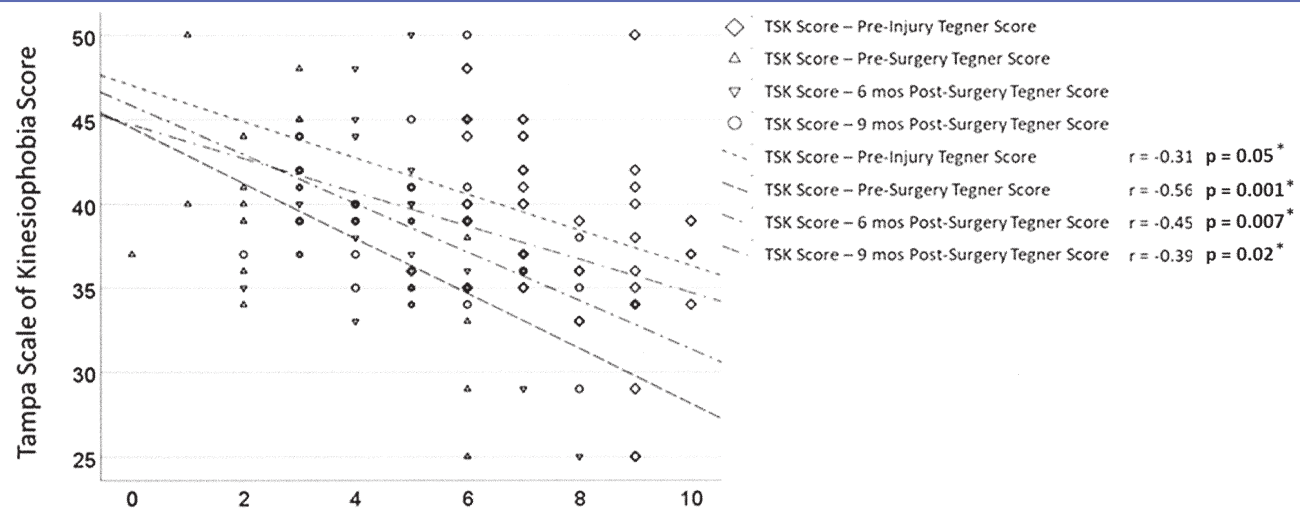

Tegner Activity Level Score

Figure 4. Significant inverse relationships existed between TSK score and Tegner Activity Level Scale scores over the 9 month study period.

TSK: Tampa Scale of Kinesiophobia; *p $\leq 0.05$.

Table II. Three month walking kinematic gait comparison.

\begin{tabular}{|c|c|c|c|c|c|}
\hline & \multicolumn{2}{|c|}{3 Month Walking } & \multicolumn{3}{|c|}{ Two-Way ANOVA } \\
\hline & $\begin{array}{l}\text { High TSK Group } \\
\text { (surgical side/non-surgical side) }\end{array}$ & $\begin{array}{l}\text { Low TSK Group } \\
\text { (surgical side/non-surgical side) }\end{array}$ & Group & Side & Group $\times$ Side \\
\hline $\begin{array}{l}\text { Hip Adduction at } \\
\text { Mid-Stance }\end{array}$ & $7.7 \pm 2.3^{\circ} / 7.1 \pm 2.3^{\circ}$ & $5.2 \pm 2.8^{\circ} / 6.6 \pm 2.4^{\circ}$ & $0.047 *$ & 0.51 & 0.45 \\
\hline $\begin{array}{l}\text { Knee Terminal } \\
\text { Stance Extension }\end{array}$ & $-31.6 \pm 11.4^{\circ} /-28.7 \pm 12.4^{\circ}$ & $-27.7 \pm 15^{\circ} /-25.5 \pm 9.1^{\circ}$ & 0.24 & 0.40 & 0.35 \\
\hline Step Length & $48.9 \pm 4.1 \mathrm{~cm} / 50.0 \pm 3.9 \mathrm{~cm}$ & $49.9 \pm 5.6 \mathrm{~cm} / 48.6 \pm 6.2 \mathrm{~cm}$ & 0.91 & 0.96 & 0.32 \\
\hline
\end{tabular}

$* \mathrm{P}<0.05$. 
Table III. Six month walking kinematic gait comparison.

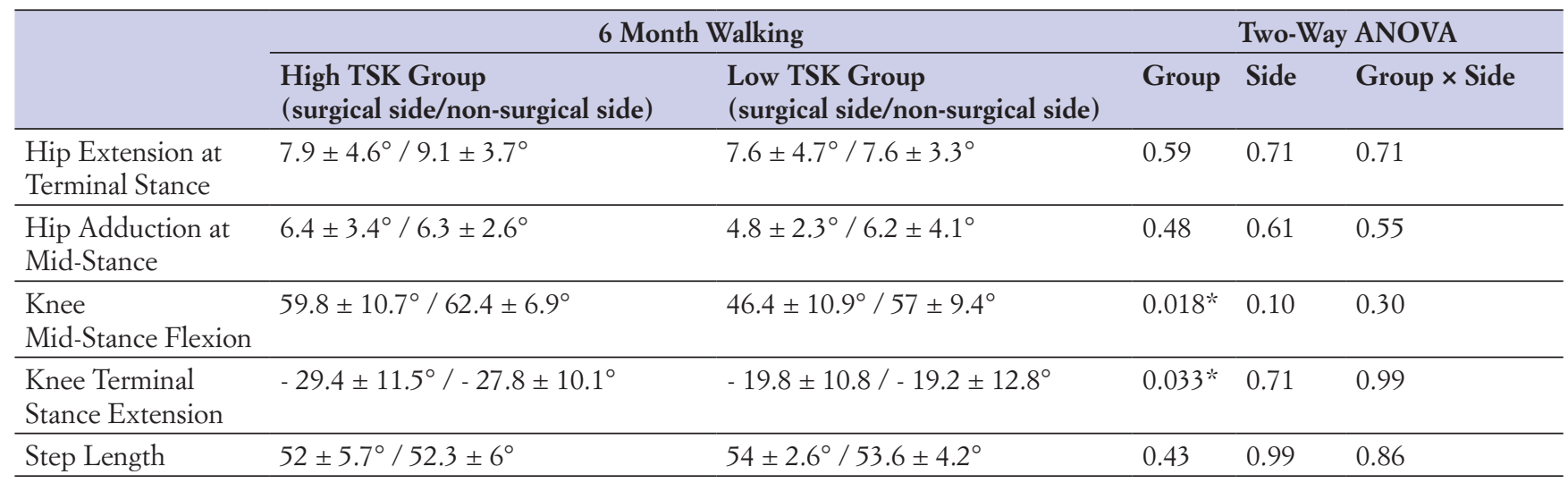

$* \mathrm{P}<0.05$.

Table IV. Six month running kinematic gait comparison.

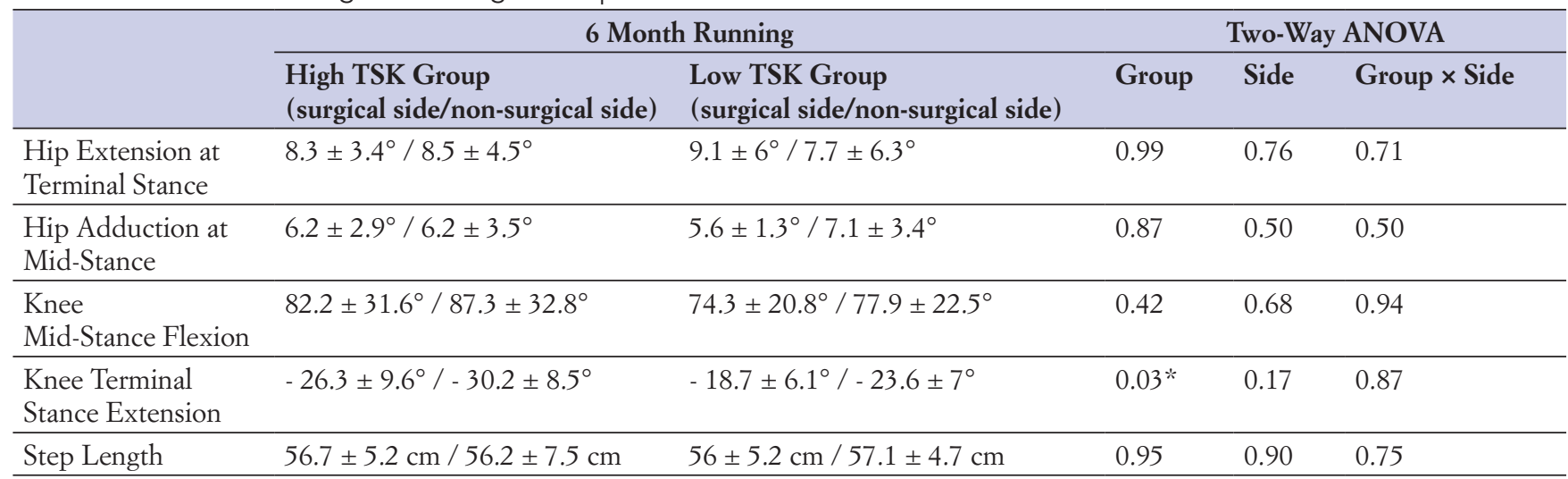

$* \mathrm{P}<0.05$.

flexion during walking than the low kinesiophobia group, but now also displayed less terminal stance phase knee extension (table III). At 6 months, the high kinesiophobia group displayed less terminal stance phase knee extension during running than the low kinesiophobia group (table IV). For each data collection period, kinesiophobia, not surgical side, was the sole factor that displayed significant kinematic gait effects.

\section{DISCUSSION}

The most important study finding was that high kinesiophobia at 3 months after ACLR (end of the acute rehabilitation phase) was related to walking and running gait kinematics at 3 and 6 months following ACLR, respectively. Kinesiophobia scores during this rehabilitation phase were also related to subject sports activity capability perceptions at 6 months and 9 months after ACLR. Among a cohort of patients that received primarily BPTB autografts, Kvist et al. (6) reported that those who had not returned to sport by 3-4 years after ACLR had greater re-injury fear. Among 73 patients who underwent ACLR using different autograft or allograft types, Lentz et al. (8) reported that regardless of graft type, patients who did not return to sport at one year following ACLR had higher kinesiophobia.

Kinesiophobia measured at 3 months after ACLR displayed a sustained inverse moderate-to-weak relationship with patient perceived sports capability before ACLR, and at 6 and 9 months following ACLR. Several studies have reported that reduced kinesiophobia is predictive of patients returning to sports at pre-injury level goals after ACLR (6-8). Despite $80 \%$ or greater surgical lower extremity hip and knee muscle strength symmetry with the non-surgical lower extremity at 4-5 months after ACLR, the current study identified a prolonged inverse relationship between the kinesiophobia measured at 3 months after ACLR (end of acute rehabilitation phase), and perceived sports activity capability measured before ACLR, and at 6 and 9 months afterwards. Increased mid-stance knee flexion when walking in the high kinesiophobia group at 3 and 6 months after ACLR may 
suggest greater functional quadriceps femoris muscle group impairment, avoidance, and/or possible re-setting of normal sagittal plane knee kinematics to protect the healing soft tissue graft. The reduced terminal stance phase knee extension observed during running in the high kinesiophobia group at 6 months after ACLR further supports this premise. At 3 months after ACLR, the high kinesiophobia group displayed greater peak stance phase hip adduction during walking than the low kinesiophobia group. This suggests greater hip abductor neuromuscular impairment with compensatory, more central surgical lower extremity foot placement to maintain lower extremity stability during early stance phase. Impaired hip muscle function is a concern following ACL injury, particular as the patient progresses to running and jumping tasks (25). Increased peak mid-stance knee flexion in the high kinesiophobia group suggests the need to prolong eccentric quadriceps femoris muscle group activation during weightbearing, possibly, to mitigate the anteriorly directed translational tibial forces that increase graft loading during terminal knee extension.

Among 12 healthy recreational runners, Orendurff et al. (26) reported that peak internal hip, knee and ankle extensor moments are sequentially distributed with peak moments occurring at 4\% (hip), 11\% (knee), and 17\% (ankle) of running stance phase. During running, peak sagittal plane knee power occurs at about $15 \%$ of stance phase with concentric-eccentric hip and knee coupling over the first $50 \%$ of stance phase. The current study suggests that peak knee extensor moments during running may occur later, and at lower magnitudes among patients with high kinesiophobia levels following ACLR. Others have identified gait compensations among patients after ACLR. Among 10 male patients of similar age, and BMI as patients in the current study, at $6.2 \pm 3.2$ months after ACLR with a hamstring autograft, Asaeda et al. (27) reported that compared to healthy subjects, patients displayed less running knee flexion and reduced internal knee extensor moments. Also, at 12 months after ACLR, maximal isokinetic knee extensor torque $\left(180^{\circ} / \mathrm{sec}\right)$, and peak knee flexion angle during running stance phase displayed a strong inverse relationship $r=-0.745$, and a strong direct relationship $r=0.83$ was observed between maximal isokinetic knee extensor torque $\left(180^{\circ} / \mathrm{sec}\right)$, and internal knee extensor moments when running. These findings support the direct relationship between increased knee flexion angle and decreased quadriceps femoris muscle group functional integrity. In comparing 12 patients at 4.5 years after ACLR with 12 healthy control subjects, Varma et al. (28) reported that during walking there was a tendency for the contralateral knee of the ACLR group to display greater peak knee flexion and lower peak internal knee extension moments compared with the ACLR knee, and both knees of the control group. They concluded that the ACLR changed bilateral knee function to reduce ACLR knee loading (28). In a study that compared 196 patients at 12 months after ACLR using BPTB autografts and 106 healthy control group subjects while walking, Davis-Wilson et al. (29) reported less knee flexion, bilaterally lower early stance phase vertical ground reaction forces, and greater midstance vertical ground reaction forces among the surgical group. Surgical group subjects also displayed lower magnitude, and earlier surgical knee stance phase flexion at 6 and 12 months after ACLR, with the non-surgical knee displaying similar characteristics by 12 months after ACLR. Compared to control group subjects, the surgical lower extremity of the ACLR group also produced lower magnitude peak stance phase internal knee extensor moments during walking at 6 and 12 months following ACLR, and the non-surgical lower extremity displayed lower magnitude peak stance phase internal knee extensor moments at 12 months after ACLR. Differences observed during walking between the non-surgical lower extremity of the ACLR group, and both lower extremities of the control group increased from 6 to 12 months postACLR. At one year after ACLR, bilateral impairments were evident, with the non-surgical lower extremity progressively resembling the surgical lower extremity, rather than the surgical lower extremity gradually returning to pre-injury characteristics (29).

Current study findings suggest that high kinesiophobia levels could potentially have a stronger influence on walking, and running lower extremity kinematic symmetry than surgical side. Based on current study findings, and the results of previous reports, biomechanical, and kinesiophobia-related compensations may develop in a manner that is somewhat unique to the patient, the rehabilitation progression, the graft that is used, and its related harvest site, and healing characteristics.

Higher TALS scores represent sport or vocational activities with progressively more intense, or more frequent knee loading challenges culminating in sports such as soccer, American football, or elite level rugby (level 10). In comparison, a TALS score of 5 represents competitive sports with lesser intensity or frequency, with more predictable knee loads such as cross-country skiing, cycling, jogging, or vocational expectations such as heavy construction labor. At the lower end of the TALS score range, level 1 represents comparatively sedentary, more desk-based work. As it increases from 1 to 10 , the TALS score represents subject perceptions of a greater ability to participate in higher level sports or vocational activities of progressively greater, and less predictable knee loads, movement ranges, sudden pivoting/directional changes, and a greater likelihood for being exposed to 
direct contact or collisions that increase the potential for further knee injury. From this perspective, the TALS score differences observed between the high and low kinesiophobia groups at each measurement period reveals how patients with seemingly comparable neuromuscular function based on current clinical assessment methods can display vastly different return to sports or vocational activity readiness perceptions. Across the entire study period, the high kinesiophobia group consistently had approximately 2-point lower perceived sports or vocational capability scores.

This study has several important limitations. The study group consisted entirely of men, therefore, these results may not generalize to women. Given the potential influence of monthly menstrual cycle on lower extremity biomechanics, connective tissue laxity, and neuromuscular control (30), the first component of this study focused exclusively on men. Although a prospective study design was used with comparable subject groups, kinematic gait comparisons were made solely between the surgical and non-surgical lower extremities of subjects who had undergone ACLR. The addition of three-dimensional kinematic, kinetic and electromyographic data may have identified more specific lower extremity impairments and compensations. Also, sagittal plane trunk position measurements may have better confirmed knee extensor function as increased forward trunk lean is known to compensate for reduced internal knee-extensor moments during running (31). Rehabilitation protocols often reference the non-surgical lower extremity when making decisions about safe return to unrestricted sports participation readiness. However, the non-surgical lower extremity may not be the best reference, as growing evidence supports bilateral neuromuscular control impairments following ipsilateral ACL injury (28). Ideally, an age, and activity-matched healthy control group would have been included in the study design. Obtaining safe return to sports readiness indices from matched healthy athletes may help establish the content and criterion validity needed to ensure that essential

\section{REFERENCES}

1. Di Stasi S, Myer GD, Hewett TE. Neuromuscular training to target deficits associated with second anterior cruciate ligament injury. J Orthop Sports Phys Ther 2013;43(11):777-92.

2. Ardern CL, Webster KE, Taylor NF, Feller JA. Return to pre-injury level of competitive sport after anterior cruciate ligament reconstructive surgery. Am J Sports Med 2011;39(3):537-43.

3. Devita P, Hortobagyi T, Barrier J. Gait biomechanics are not normal after anterior cruciate ligament reconstruction and accelerated rehabilitation. Med Sci Sports Exerc 1998;30(10):1481-8. neuromuscular, and psychobehavioral recovery thresholds have been achieved. Lastly, study duration was only over the initial 9 months following ACLR. Recent reports suggest that functional recovery after ACLR may take upwards of 2 years (32). The study time period was selected to coincide with the rehabilitation program phases used at our institution which generally culminate with return to sports at 9 months following ACLR.

\section{CONCLUSIONS}

High kinesiophobia at 3 months after ACLR was related to walking and running gait kinematic characteristics 3 and 6 months post-surgery, respectively. They were also related to subject perceptions of sports activity capability at 6 months and 9 months after ACLR.

\section{Main points}

- Tegner Activity Level Scores were similar between groups prior to the index knee injury, however, the high kinesiophobia group had lower scores pre-surgery, and at 6 and 9 months after ACLR.

- By 9 months after ACLR, kinesiophobia continued to influence perceived sports capability.

- Kinesiophobia had a stronger influence on kinematic gait patterns than surgical versus non-surgical lower extremity differences.

\section{FUNDINGS}

This study was supported by a grant from the SingHealth Academic Medical Centre.

\section{CONFLICT OF INTERESTS}

The authors declare that they have no conflict of interests.

4. Knoll Z, Kiss RM, Kocsis L. Gait adaptation in ACL deficient patients before and after anterior cruciate ligament reconstruction surgery. J Electromyogr Kinesiol 2004;14(3):287-94.

5. Butler RJ, Minick KI, Ferber R, Underwood F. Gait mechanics after ACL reconstruction: implications for the early onset of knee osteoarthritis. Br J Sports Med 2009;43(5):366-70.

6. Kvist J, Ek A, Sporrstedt K, Good L. Fear of re-injury: a hindrance for returning to sports after anterior cruciate ligament reconstruction. Knee Surg Sports Traumatol Arthrosc 2005;13(5):393-7.

7. Chmielewski TL, Jones D, Day T, Tillman SM, Lentz TA, George SZ. The association of pain and fear of movement/ 
reinjury with function during anterior cruciate ligament reconstruction rehabilitation. J Orthop Sports Phys Ther 2008;38(12):746-53.

8. Lentz TA, Zeppieri Jr G, George SZ, Tillman SM, et al. Comparison of physical impairment, functional, and psychosocial measures based on fear of reinjury/lack of confidence and return-to-sport status after ACL reconstruction. Am J Sports Med 2015;43(2):345-53.

9. Ericksson YB, Roos EM, Frobell RB. Lower extremity performance following ACL rehabilitation in the Kanon Trial: impact of reconstruction and predictive values at 2 and 5 years. $\mathrm{Br} \mathrm{J}$ Sports Med 2013;47(15):980-5.

10. Paterno MV, Schmitt LC, Ford KR, et al. Biomechanical measures during landing and postural stability predict second anterior cruciate ligament injury after anterior cruciate ligament reconstruction and return to sport. Am J Sports Med 2010;38:1968-78.

11. Nagelli CV, Hewett TE. Should return to sport be delayed until two years after anterior cruciate ligament reconstruction? Biological and functional considerations. Sports Med 2017;47(2):221-32.

12. Nyland J, Greene J, Carter S, Brey J, Krupp R, Caborn D. Return to sports bridge program improves outcomes, decreases ipsilateral knee re-injury and contralateral knee injury rates post-ACL reconstruction. Knee Surg Sports Traumatol Arthrosc 2020;28(11):3676-85.

13. Miller RP, Kori S, Todd D. The Tampa Scale: a measure of kinesiophobia. Clin J Pain 1991;7(1):51-2.

14. Pool J, Hiralal S, Ostelo R, et al. The applicability of the Tampa Scale of Kinesiophobia for patients with sub-acute neck pain: a qualitative study. Qual Quant 2009;43:773-80.

15. Vlaeyen JW, Crombez G, Linton SJ. The fear-avoidance model of pain. Pain 2016;157(8):1588-9.

16. Tegner Y, Lysholm J. Rating systems in the evaluation of knee ligament injuries. Clin Orthop Relat Res 1985;198:43-9.

17. Lysholm J, Gillquist J. Evaluation of knee ligament surgery results with special emphasis on use of a scoring scale. Am J Sports Med 1982;10(3):150-4.

18. Briggs KK, Lysholm J, Tegner Y, Rodkey WG, Kocher MS, Steadman JR. The reliability, validity, and responsiveness of the Lysholm score and Tegner Activity Scale for anterior cruciate ligament injuries of the knee: 25 years later. Am J Sports Med 2009;37(5):890-7.

19. Nyland J, Mattocks A, Kibbe S, Kalloub A, Greene JW, Caborn DN. Anterior cruciate ligament reconstruction, rehabilitation, and return to play: 2015 update. Open Access J Sports Med 2016;7:21-32.
20. Nyland J, Brand E, Fisher B. Update on rehabilitation following ACL reconstruction. Open Access J Sports Med 2010;1:151-66.

21. Cronin J, Nash M, Whatman C. Assessing dynamic knee joint range of motion using siliconcoach. Phys Ther Sport 2006;7(4):191-4.

22. McDonald DA, Delgadillo JQ, Fredericson M, McConnell J, Hodgins M, Besier TF. Reliability and accuracy of a video analysis protocol to assess core ability. PM\&R 2011;3(3):204-11.

23. Weir G, Alderson J, Smailes N, Elliott B, Donnelly C. A reliable video-based ACL injury screening tool for female team sport athletes. Int J Sports Med 2019;40(3):191-9.

24. Mukaka MM. Statistics corner: A guide to appropriate use of correlation coefficient in medical research. Malawi Med J 2012;24(3):69-71.

25. Kline PW, Burnham J, Yonz M, Johnson D, Ireland ML, Noehren B. Hip external rotation strength predicts hop performance after anterior cruciate ligament reconstruction. Knee Surg Sports Traumatol Arthrosc 2018;26(4):1137-44.

26. Orendurff MS, Kobayashi T, Tulchin-Francis K, et al. A little bit faster: Lower extremity joint kinematics and kinetics as recreational runners achieve faster speeds. J Biomech 2018;71:167-75.

27. Asaeda M, Deie M, Kono Y, Mikami Y, Kimura H, Adachi $\mathrm{N}$. The relationship between knee muscle strength and knee biomechanics during running at 6 and 12 months after anterior cruciate ligament reconstruction. AP-SMART 2019;16:14-8.

28. Varma RK, Duffell LD, Nathwani D, McGregor AH. Knee moments of anterior cruciate ligament reconstructed and control participants during normal and inclined walking. BMJ Open 2014;4:e004753.

29. Davis-Wilson HC, Pfeiffer SJ, Johnston CD, et al. Bilateral gait 6 and 12 months post-anterior cruciate ligament reconstruction compared with controls. Med Sci Sports Exerc 2020;52:785-94.

30. Balachandar V, Marciniak J-L, Wall O, Balachandar C. Effects of the menstrual cycle on lower-limb biomechanics, neuromuscular control, and anterior cruciate ligament injury risk: A systematic review. Muscles Ligaments Tendons J 2017;7(1):136-46.

31. Teng H-L, Powers CM. Hip-extensor strength, trunk posture, and use of the knee-extensor muscles during running. J Athl Train 2016;51(7):519-24.

32. Nagelli CV, Hewett TE. Should return to sport be delayed until 2 years after anterior cruciate ligament reconstruction? Biological and functional considerations. Sports Med 2017;47(2):221-32. 